\title{
Production data of wine grape gene bank (Vitis spp.) of University of Debrecen, east Hungary
}

\author{
Rakonczás, $\mathbf{N}$. \\ University of Debrecen, Faculty of the Agricultural and Food Sciences and Environmental Management, \\ Institute of Horticulture, 138. Böszörményi str., Debrecen, H-4032, Hungary \\ Author for correspondence: rakonczas@agr.unideb.hu
}

\begin{abstract}
Summary: National wine strategy of Hungary promotes the use of "flexible" grape cultivars. These enable producers' best fit to wine market changes and expectations. This study is aimed to present data on the gene bank of the University of Debrecen, Hungary. Data were collected at a single site, between 2010 and 2018 in east Hungary lowland on acidic sandy soil, own rooted planting material. Our results showed that besides high yield and adequate cane production desired sugar content at convenient $\mathrm{pH}$ is to be awaited with moderate deviation between vintages. Presented concept demonstrate technological flexibility of cultivars by their average deviation from regression equation between increasing sugar and $\mathrm{pH}$ typical for the vintage composed of data of cultivars of the gene bank. Average positive deviation means higher sugar content at specific $\mathrm{pH}$, thus higher sugar content at desired, conveniently low $\mathrm{pH}(3.0-3.2 \mathrm{pH})$.
\end{abstract}

Rakonczás, N. (2019): Production data of wine grape gene bank (Vitis spp.) of University of Debrecen, east Hungary. International Journal of Horticultural Science 25(3-4): 32-36. https://doi.org/10.31421/IJHS/25/3-4/4943

Key words: wine grape, yield, cane, $\mathrm{pH}$, sugar, regression

\section{Introduction}

National wine strategy points out quality wine production. This is the strength of Hungarian wine production sector. Mass production is not an option in international market concern. Another aim of the strategy to focus on "flexible" cultivars. This means that a circle of cultivars could be defined technologically flexible rendering possibilities for growers and wine makers to better fit to wine-market (for example: 'Kékfrankos', 'Rizling', 'Furmint'). Production goal can greatly vary, but there is a general need for a portfolio of products basing financial safety of the enterprise. Possibilities could be backed by data on biological productivity potential of the cultivars. This paper is to disseminate data of the variety collection of University of Debrecen from this perspective.

Earlier papers focusing on resistant, interspecific (PIWI) cultivars (Rakonczás, 2011, 2015) detail technological possibilities and strength of this innovative group. The importance of these varieties (and hybrids) is considerably increasing due to very expansive effects of globalization and climate change. These highly affect vine growing- and wine making technologies. Producers are increasingly forced to focus on the market. Biological potential and resistance to pests and climatic extremities, thus also ecological adaptability of cultivars became crucial information.

Biological productivity of cultivars could be primitively demonstrated with average yearly harvest data. Ravaz-index encomprise data of yearly cane production (Ravaz, 1903). There is a general rule, that higher quantity equals lower quality. It is important to comprehend, that this rule in this wording is very rough. System of sink-source relations is more sophisticated and highly more sensitive with its physiological background (Csepregi, 1982, Ribereau et al., 2006, Keller, 2000, Lörincz \& Barócsi, 2010), and market expectations (problematics of rosé wine).
Ravaz-index (Ravaz, 1903) can greatly deviate, according to the vegetative or generative character of the variety, to applied phytotechnical practices and the condition of the plantation. It is important to respect that the derived data hides the real total biological productivity $(10 \mathrm{~kg} / 5 \mathrm{~kg}=2$ and $2 \mathrm{~kg} / 1 \mathrm{~kg}=2$ ).

In this context Tomcsányi \& Német (1963) cit. Csepregi (1982) state that a variety is to be deemed valuable, of which the cane production between vintages does not show great variation, and in comparison to other cultivars a considerably higher yield is harvested on the same level of cane production. A criteria is to be added: besides higher and stable yield with adequate and stable cane production (Német, 1963, Csepregi, 1982) the desired sugar content is reached besides adequately low pH (Rakonczás, 2015).

\section{Materials and methods}

The variety collection of the University of Debrecen was established in Pallag, on immune sandy soil, by $3 \mathrm{~m}$ between row and $1 \mathrm{~m}$ between stock spacing trained for single curtain stock form, with the use of European own rooted planting material. Five stocks of each cultivar represent one experimental block.

Nutrition is carried out on the basis of the specific nutrient demand of the grape (Kozma, 1993) giving out $310 \mathrm{~kg} \mathrm{NPK}$ (effective material) manure on yearly bases in two phases. More detailed description was given in an earlier paper (Rakonczas, 2015). Because of considerable presence of ESCA, this work represents our last published data collection in this topic.

The following data were collected: yield (kg/stock), cane production $(\mathrm{kg} / \mathrm{stock})$, of which the (Ravaz-index, or Y/N-ratio 
(Ravaz, 1903)) the use-up index of wood yield is calculated (kg yield/kg cane). Sugar content and acidity at harvest is presented according to the introduced concept.

Data were processed with Microsoft Excel. Besides average data of 2010-2018, CV is also given: $\mathrm{CV}=(($ Deviation / Average of vintages) * 100). This is to demonstrate technological-, climatic robustness of the cultivars.

\section{Results and discussion}

It is clear to comprehend that besides adequate yield and cane production, better technological flexibility of the variety could be detected in phenomena, when increasing sugar content meats lower level of acidic breakdown (less steep $\mathrm{pH}$ increase) (Table 1). By increasing sugar content at ripening, $\mathrm{pH}$ consequently increases, since titratable acidity lowers (Marcus, 2000; Ribéreau et al., 2006). On average, producer could estimate an increase of about 1.5-2.0 sugar degree a weak. In optimal case 18-20 Hungarian sugar degree (180-200 $\mathrm{g} /$ litre) is reached at $3.0-3.2 \mathrm{pH}$. Within variety categories in each vintage regression equation was put on data of varieties of the collection. Deviation of single variety data from regression line shows, whether sugar accumulation of specific cultivars at a given $\mathrm{pH}$ is lower or higher than the average. The best case is to get higher sugar degree at lower $\mathrm{pH}$, in case of corresponding high yield and adequate cane production. This demonstration does not content data of 2014 for abnormal rainfall and berry rot, and 2016 for problems of $\mathrm{pH}$ measurement. In case of red wines varieties 2016 is also missing, because few data could be collected.

Table 1. Regression equations of ripening parameters for white and red wine grapes (Debrecen - Pallag, 2012-2018)

\begin{tabular}{|l|c|l|}
\hline \multicolumn{3}{|l|}{ White wine cultivars: } \\
\hline 2012 & $\mathrm{y}=0.764 \mathrm{x}+18.333$ & $\mathrm{R}^{2}=0.0065$ \\
\hline 2013 & $\mathrm{y}=7.8709 \mathrm{x}+3.7009$ & $\mathrm{R}^{2}=0.2653$ \\
\hline 2015 & $\mathrm{y}=4.8582 \mathrm{x}+4.2051$ & $\mathrm{R}^{2}=0.0798$ \\
\hline 2017 & $\mathrm{y}=0.6854 \mathrm{x}+20.78$ & $\mathrm{R}^{2}=0.0042$ \\
\hline 2018 & $\mathrm{y}=0.0983 \mathrm{x}+17.926$ & $\mathrm{R}^{2}=0.3357$ \\
\hline Red wine cultivars: & $\mathrm{y}=3.1101 \mathrm{x}+11.018$ & $\mathrm{R}^{2}=0.0804$ \\
\hline 2012 & $\mathrm{y}=8.1571 \mathrm{x}-8.4087$ & $\mathrm{R}^{2}=0.3046$ \\
\hline 2015 & $\mathrm{y}=5.38 \mathrm{x}+2.7022$ & $\mathrm{R}^{2}=0.2764$ \\
\hline 2017 & $\mathrm{y}=5.2918 \mathrm{x}+3.2033$ & $\mathrm{R}^{2}=0.0957$ \\
\hline 2018 & \multicolumn{2}{|c|}{} \\
\hline
\end{tabular}

From the point of mass production the following white wine grape cultivars are to be highlighted (Tables 2-3): 'Cirfandli', 'Generosa', 'Aletta', 'Göcseji zamatos' (high yield and cane production). It is to be mentioned, that nearly all interspecific varieties show higher values both in yield and cane production. From the point of discussed concept, these data demonstrate the vigour and production potential of specific cultivars.

On second step, deviation of sugar accumulation compared to regression equation line at a given $\mathrm{pH}$ (decision of wine making technology) demonstrate adaptability of the specific variety to the concept of technological flexibility. Levels of CV mark between-vintage stability of the data (the lower the better). Presented data suggest, that qualified 'Ezerfürtü',
'Generosa', 'Korona', 'Pátria', 'Sauvignon', 'Zengő', 'Zéta', 'Zeus'; from the circle of candidates 'Gyöngyrizling', 'Heureka' and 'Tarcal 1 and 7' represent perspective choice. From the circle of interspecific (PIWI, or innovative, resistant group) cultivars 'Kunleány', 'Odysseus' and 'Viktoria gyöngye' show perspective data.

Set of production data of red wine grape cultivars does not comprise any extending case (Table 4$)$. Cane production does not show in-balanced vegetative-generative harmony either. It is due to note, that other type of pruning and training strategy instead of single curtain - would probably facilitate higher production levels. Only 'Cabernet sauvignon', 'Alicante Bouchet', 'CsV525', 'Dornfelder' and interspecific 'Duna gyöngye' (Perla de Danube) could probably be highlighted.

Concept of flexibility can not be shown in case of red wine cultivars, because data bias (ESCA, etc.) and inconveniences of the training system. Data of cultivars of good reputation like 'Kékfrankos' (Blaufrankish) gives an example for this constraint.

\section{Conclusions}

The presented concept could give a guideline in evaluation of wine grape cultivars. In integrated production each element of the technology should aim at production policy, and support marketing. Plant protection and plant condition (capacity and health of tissues) is also essential in quality wine production besides discussed production data and basic quality parameters. In an ideal plant condition pattern and structure of the plantation, besides appropriate training system and pruning strategy, modification of technological elements - most of all load of the stacks, fitotechnical measures (Lörincz \& Barócsi, 2010) - considerable flexibility can be achieved. Highlighted cultivars of this paper give suggestion to chose the best appropriate cultivars of better flexibility.

\section{References}

Csepregi P. (1982): A szőlő metszése, fitotechnikai műveletei. Mezőgazdasági Kiadó, Budapest. 357. p.

Keller, M. (2010): The Science of Grapevines: Anatomy and Physiology. Academic Press; 508. p.

Lőrincz, A., Barócsi, Z. (2010): A szőlő metszése és zöldmunkái. Mezőgazda Kiadó, Budapest. 306. p.

Rakonczás, N. (2011): Mostoha gyermekeink, Rezisztens szőlőfajták. Bor és Piac, 5-6. 14-17. p.

Rakonczás, N. (2015): Biological performance of grape cultivars in Eastern Hungary. International Journal of Horticultural Science, 1-2. 31-36. p.

Ravaz, L. (1903) Sur la brunissure de la vigne. Les Comptes Rendus de l'Académie des Sciences, 136. 1276-1278. p.

Ribéreau-Gayon, P., Dubourdieu, D., Doneche, B., Lonvaud, A. (2006): Handbook of Enology, Vol. 1: The Microbiology of Wine and Vinifications. Wiley. 497. p.

Tomcsányi P., Németh L. (1963): A szőlőtermés elemzése és növekedése. Nemesített növényfajtákkal végzett országos fajtakísérletek eredményei. 417-948. p. 
Table 2. Production and flexibility data of qualified Vitis vinifera (L.) white wine grape varieties (Pallag, 2010-2018).

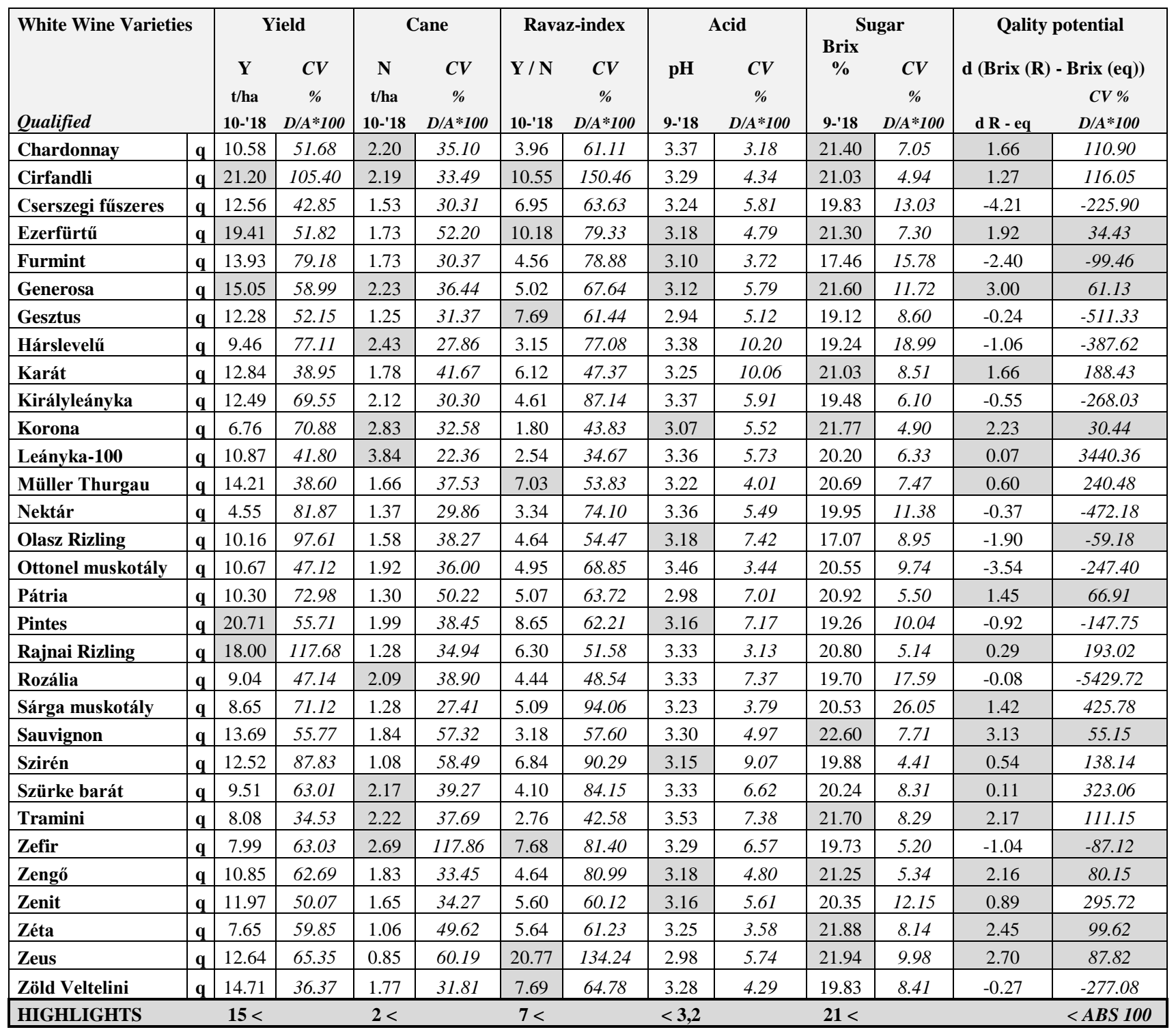


Table 3. Production and flexibility data of candidate Vitis vinifera (L.) and intespecific (qualified and candidate) white wine grape varieties (Pallag, 2010-2018).

\begin{tabular}{|c|c|c|c|c|c|c|c|c|c|c|c|c|c|}
\hline \multirow{4}{*}{\multicolumn{2}{|c|}{$\begin{array}{l}\text { White Wine Varieties } \\
\text { Candidates }\end{array}$}} & \multirow{2}{*}{\multicolumn{2}{|c|}{ Yield }} & \multirow{2}{*}{\multicolumn{2}{|c|}{ Cane }} & \multirow{2}{*}{\multicolumn{2}{|c|}{ Ravaz-index }} & \multirow{2}{*}{\multicolumn{2}{|c|}{ Acid }} & \multirow{2}{*}{\multicolumn{2}{|c|}{ Sugar }} & \multirow{3}{*}{\multicolumn{2}{|c|}{$\begin{array}{r}\text { Qality potential } \\
\text { d (Brix (R) - Brix (eq)) } \\
\text { CV \% }\end{array}$}} \\
\hline & & & & & & & & & & & & & \\
\hline & & \multirow{3}{*}{ 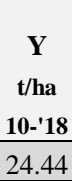 } & \multirow{2}{*}{$\begin{array}{c}C V \\
\% \\
D / A * 100 \\
\end{array}$} & \multirow{3}{*}{$\begin{array}{c}\begin{array}{c}\mathbf{N} \\
\text { t/ha } \\
10-' 18\end{array} \\
1.48\end{array}$} & \multirow{2}{*}{$\begin{array}{c}C V \\
\% \\
D / A * 100 \\
\end{array}$} & \multirow{3}{*}{$\begin{array}{l}\mathrm{Y} / \mathrm{N} \\
10-' 18 \\
1231\end{array}$} & \multirow{2}{*}{$\begin{array}{c}C V \\
\% \\
D / A * 100 \\
\end{array}$} & \multirow{3}{*}{$\begin{array}{c}\text { pH } \\
\text { 9-'18 } \\
3.10\end{array}$} & \multirow{2}{*}{$\begin{array}{c}C V \\
\% \\
D / A * 100 \\
\end{array}$} & \multirow{2}{*}{$\begin{array}{c}\text { Brix } \\
\% \\
9-' 18 \\
\end{array}$} & \multirow{2}{*}{$\begin{array}{c}C V \\
\% \\
D / A * 100 \\
\end{array}$} & & \\
\hline & & & & & & & & & & & & d R - eq & $D / A * 100$ \\
\hline B-11 & can & & 42.57 & & 24.15 & & 61.73 & & 4.69 & 17.72 & 9.75 & -0.65 & -154.81 \\
\hline C-28 & can & 7.82 & 57.64 & 2.95 & 54.37 & 3.10 & 92.04 & 3.44 & 3.81 & 20.45 & 2.17 & -0.91 & -59.96 \\
\hline Calábriai fehér & can & 6.76 & 76.80 & 3.04 & 67.31 & 2.40 & 85.45 & 3.32 & 5.58 & 18.30 & 5.21 & -1.77 & -37.01 \\
\hline CSFT-92 & can & 14.61 & 101.77 & 2.01 & 41.21 & 5.50 & 87.00 & 3.31 & $*$ & 17.00 & $*$ & -1.93 & -141.42 \\
\hline Csiri-Csuri & can & 16.66 & 136.78 & 1.50 & 34.45 & 3.76 & 60.76 & 3.28 & 2.33 & 18.50 & 9.76 & $\begin{array}{l}-1.69 \\
\end{array}$ & -63.56 \\
\hline CSVT-47 & can & 10.08 & 79.29 & 1.81 & 48.50 & 3.15 & 68.71 & 3.34 & 2.89 & 20.78 & 8.90 & -1.08 & -239.77 \\
\hline Gyöngy rizling & can & 8.41 & 47.69 & 1.51 & 33.81 & 5.40 & 57.71 & 3.59 & 1.97 & 23.30 & 13.81 & 2.44 & 78.58 \\
\hline Heuréka & can & 13.12 & 61.60 & 1.01 & 61.02 & 13.06 & 78.45 & 2.97 & 4.24 & 21.66 & 7.26 & 1.79 & 53.63 \\
\hline Jubileum 75 & can & 13.09 & 43.76 & 1.73 & 49.14 & 7.20 & 58.91 & 3.24 & 5.01 & 19.39 & 11.02 & 0.01 & 21480.31 \\
\hline Jubileum srébe & can & 17.61 & 185.08 & 0.88 & 32.70 & 4.11 & 98.77 & 3.54 & 6.03 & 22.02 & 10.30 & 2.27 & 67.71 \\
\hline Kecskemét virága & can & 14.64 & 53.24 & 1.12 & 41.40 & 10.78 & 73.86 & 3.44 & 7.47 & 16.97 & 11.94 & -3.91 & -48.01 \\
\hline Kecskemét-13 & can & 16.55 & 75.24 & 1.40 & 30.75 & 7.72 & 66.59 & 3.06 & 5.86 & 17.72 & 13.30 & -1.65 & -98.18 \\
\hline Mátrai muskotály & can & 17.94 & 57.13 & 1.90 & 56.49 & 6.61 & 70.41 & 3.27 & 2.78 & 18.98 & 7.04 & -1.69 & -75.58 \\
\hline Muscat bouche & can & 11.71 & 116.33 & 2.43 & 38.49 & 2.49 & 67.98 & 3.42 & 7.52 & 18.64 & 8.40 & -1.67 & -97.27 \\
\hline Nosztori Rizling & can & 9.97 & 58.20 & 1.18 & 27.75 & 6.92 & 87.83 & 3.24 & 6.08 & 19.22 & 5.25 & -0.43 & -484.13 \\
\hline Tarcal-1 & can & 7.59 & 46.09 & 0.85 & 65.54 & 5.60 & 105.06 & 3.50 & 0.81 & 23.60 & 5.99 & 3.81 & 93.26 \\
\hline Tarcal-15 & can & 8.81 & 98.88 & 1.05 & 78.75 & 11.05 & 160.28 & 3.44 & 5.58 & 21.06 & 6.05 & 0.57 & 330.97 \\
\hline Tarcal-3 & can & 13.36 & 60.05 & 2.13 & 16.59 & 4.04 & 60.57 & 3.10 & 6.11 & 20.26 & 4.90 & 0.68 & 195.05 \\
\hline Tarcal-41 & can & 8.81 & 50.63 & 1.37 & 40.91 & 3.88 & 56.78 & 3.43 & 4.46 & 20.48 & 16.53 & -0.78 & -271.57 \\
\hline Tarcal-7 & can & 12.34 & 79.08 & 2.56 & 23.87 & 2.28 & 38.19 & 3.29 & 4.62 & 21.84 & 9.68 & 2.83 & 94.30 \\
\hline Tarcal-8 & can & 9.63 & 46.80 & 0.83 & 26.26 & 10.03 & 73.54 & 3.28 & 6.89 & 21.55 & 5.61 & 1.92 & 127.54 \\
\hline HIGHLIGHTS & & $15<$ & & $2<$ & & $7<$ & & $<3.2$ & & $21<$ & & & $<A B S 100$ \\
\hline
\end{tabular}

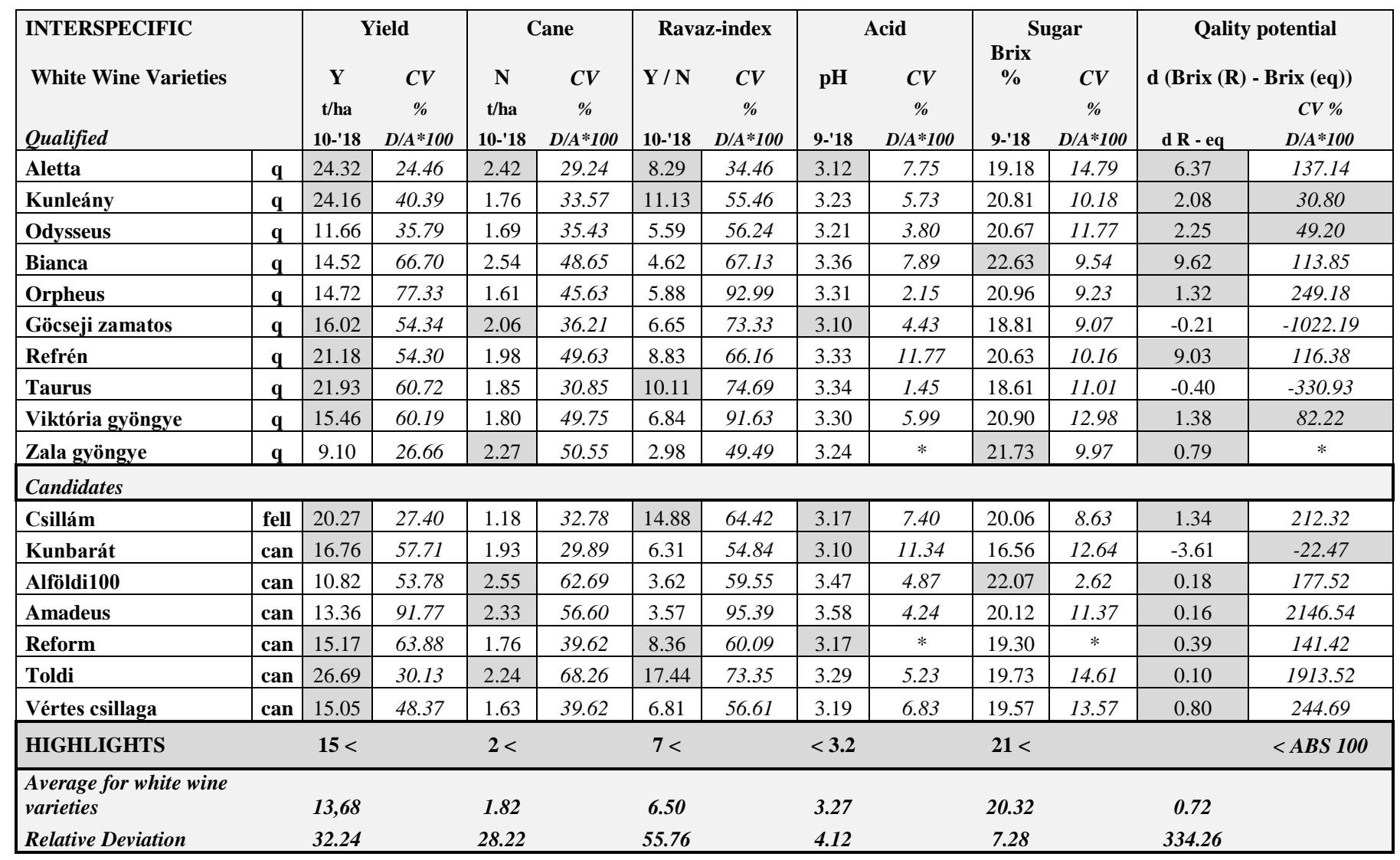


Table 4. Production and flexibility data of red wine grape varieties (Pallag, 2010-2018).

\begin{tabular}{|c|c|c|c|c|c|c|c|c|c|c|c|c|c|}
\hline \multicolumn{2}{|l|}{ Red Wine Varieties } & \multicolumn{2}{|c|}{ Yield } & \multicolumn{2}{|c|}{ Cane } & \multicolumn{2}{|c|}{ Ravaz-index } & \multicolumn{2}{|c|}{ Acid } & \multicolumn{2}{|c|}{ Sugar } & \multirow{2}{*}{\multicolumn{2}{|c|}{$\begin{array}{r}\text { Qality potential } \\
\text { d (Brix (R) - Brix (eq)) } \\
\text { CV \% }\end{array}$}} \\
\hline & & \multirow{3}{*}{ 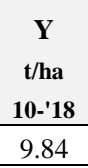 } & \multirow{2}{*}{$\begin{array}{c}C V \\
\% \\
D / A * 100 \\
\end{array}$} & \multirow{3}{*}{$\begin{array}{c}\begin{array}{c}N \\
\text { t/ha } \\
10-' 18\end{array} \\
3.00 \\
\end{array}$} & \multirow{2}{*}{$\begin{array}{c}C V \\
\% \\
D / A * 100 \\
\end{array}$} & \multirow{3}{*}{$\begin{array}{l}\mathrm{Y} / \mathrm{N} \\
10-' 18 \\
268\end{array}$} & \multirow{2}{*}{$\begin{array}{c}C V \\
\% \\
D / A * 100 \\
\end{array}$} & \multirow{2}{*}{$\begin{array}{l}\text { pH } \\
9-' 18 \\
\end{array}$} & \multirow{2}{*}{$\begin{array}{c}C V \\
\% \\
D / A * 100 \\
\end{array}$} & \multirow{2}{*}{$\begin{array}{l}\text { Brix } \\
\% \\
9-' 18 \\
\end{array}$} & \multirow{2}{*}{\begin{tabular}{c|}
$C V$ \\
$\%$ \\
$D / A * 100$ \\
\end{tabular}} & & \\
\hline Qualified & & & & & & & & & & & & d R - eq & $D / A * 100$ \\
\hline Bíbor kadarka & $\mathbf{q}$ & & 78.26 & & 32.08 & & 60.38 & 3.29 & 5.68 & 19.83 & 25.50 & 1.59 & 221.56 \\
\hline Cabernet Franc & $q$ & 10.88 & 57.92 & 2.56 & 36.13 & 3.99 & 93.16 & 3.14 & 4.71 & 20.62 & 5.34 & 0.82 & 227.22 \\
\hline Cabernet Sauvignon & $q$ & 14.47 & 91.59 & 2.20 & 30.04 & 3.92 & 70.21 & 3.03 & 7.90 & 17.39 & 36.85 & 1.25 & 137.45 \\
\hline Dornfelder & $q$ & 13.74 & 102.78 & 2.91 & 85.12 & 3.93 & 67.18 & 3.50 & 4.37 & 19.66 & 2.98 & -1.94 & -31.57 \\
\hline Kadarka & $q$ & 2.89 & 89.42 & 3.02 & 41.74 & 1.04 & 114.01 & 3.25 & 4.77 & 18.43 & 7.83 & -2.73 & -32.64 \\
\hline Kármin & $q$ & 8.61 & 100.31 & 1.62 & 38.76 & 3.91 & 93.89 & 3.33 & 4.52 & 19.84 & 10.66 & 0.07 & 2767.53 \\
\hline Kékfrankos & $q$ & 9.14 & 65.19 & 1.46 & 21.42 & 5.70 & 85.77 & 3.12 & 6.51 & 17.53 & 11.31 & -1.42 & -164.48 \\
\hline Kékoportó & $q$ & 9.10 & 103.60 & 1.45 & 26.91 & 3.58 & 99.42 & 3.57 & 0.28 & 21.10 & 13.69 & 0.00 & -117892.71 \\
\hline Merlot & $q$ & 9.46 & 67.70 & 2.32 & 70.90 & 4.29 & 64.11 & 3.26 & 5.10 & 19.86 & 7.33 & 0.69 & 240.24 \\
\hline Pinot Noir & $q$ & 11.55 & 94.95 & 1.79 & 31.09 & 5.20 & 98.93 & 3.22 & 5.43 & 20.86 & 9.27 & 0.81 & 327.51 \\
\hline Zweigelt & $q$ & 7.40 & 70.20 & 1.42 & 25.98 & 4.92 & 83.61 & 3.15 & 2.95 & 18.85 & 4.68 & -1.86 & $*$ \\
\hline \multicolumn{14}{|l|}{ Candidates } \\
\hline Alicante Bouschet & can & 19.31 & 72.84 & 2.89 & 80.83 & 5.13 & 79.02 & 3.27 & 6.64 & 17.46 & 13.05 & -2.34 & -4.83 \\
\hline CSV-420 & can & 8.82 & 85.37 & 1.82 & 29.34 & 5.88 & 118.60 & 3.34 & 2.14 & 20.10 & 7.46 & 0.43 & 106.99 \\
\hline CSV-525 & can & 12.70 & 47.18 & 2.02 & 22.99 & 5.26 & 81.70 & 3.31 & 4.08 & 20.90 & 4.13 & 0.95 & 61.27 \\
\hline Kurucvér & can & 7.93 & 54.19 & 2.86 & 38.30 & 2.34 & 46.53 & 3.42 & 4.91 & 21.50 & 7.94 & 0.03 & 6039.08 \\
\hline Magyar frankos & can & 12.20 & 88.79 & 1.83 & 48.45 & 3.96 & 87.54 & 3.20 & 5.87 & 20.08 & 5.00 & 0.19 & 344.88 \\
\hline Miklóstelep 7 & can & 7.01 & 107.99 & 1.32 & 40.95 & 4.12 & 183.49 & 3.41 & 5.32 & 21.13 & 15.91 & 0.42 & 833.57 \\
\hline Rubintos & can & 11.68 & 53.14 & 1.38 & 30.40 & 6.51 & 63.59 & 3.08 & 5.97 & 19.54 & 9.23 & 0.79 & 35.70 \\
\hline
\end{tabular}

\begin{tabular}{|c|c|c|c|c|c|c|c|c|c|c|c|c|c|}
\hline \multicolumn{2}{|l|}{$\begin{array}{l}\text { INTERSPECIFIC } \\
\text { Red Wine Varieties } \\
\text { Qualified }\end{array}$} & \multirow{2}{*}{$\begin{array}{c}\begin{array}{c}Y \\
\text { t/ha } \\
10-' 18\end{array} \\
3.44 \\
\end{array}$} & $\begin{array}{c}C V \\
\% \\
D / A * 100 \\
\end{array}$ & \multirow{2}{*}{ 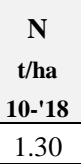 } & $\begin{array}{c}C V \\
\% \\
D / A * 100\end{array}$ & $\begin{array}{r}Y / N \\
10-' 18 \\
\end{array}$ & $\begin{array}{c}C V \\
\% \\
D / A * 100 \\
\end{array}$ & $\begin{array}{c}\text { pH } \\
9-' 18\end{array}$ & $\begin{array}{c}C V \\
\% \\
D / A * 100 \\
\end{array}$ & $\begin{array}{l}\text { Brix } \\
\% \\
9-' 18\end{array}$ & $\begin{array}{c}C V \\
\% \\
D / A * 100 \\
\end{array}$ & \multicolumn{2}{|c|}{$\begin{array}{r}\text { Qality potential } \\
\text { d (Brix (R) - Brix (eq)) } \\
\text { CV \% }\end{array}$} \\
\hline Korai bíbor & $q$ & & 144.74 & & 26.95 & 2.33 & 134.06 & 3.34 & 2.72 & 24.73 & 9.75 & 3.95 & 60.62 \\
\hline Medina & $q$ & 9.25 & 51.30 & 1.52 & 48.30 & 6.26 & 46.03 & 3.29 & 10.32 & 20.60 & 9.90 & -0.75 & -160.81 \\
\hline Pannon frankos & $q$ & 13.80 & 58.36 & 1.72 & 28.24 & 7.14 & 63.93 & 3.32 & 8.43 & 20.50 & 20.69 & 1.30 & 62.53 \\
\hline Turán & $q$ & 8.27 & 88.54 & 1.97 & 41.30 & 3.33 & 73.69 & 3.62 & 4.43 & 21.87 & 2.76 & -0.53 & -41.12 \\
\hline \multicolumn{14}{|l|}{ Candidates } \\
\hline Duna Gyöngye & can & 16.87 & 70.14 & 1.26 & 42.32 & 9.69 & 78.48 & 3.48 & 5.92 & 19.60 & 11.00 & -0.48 & -209.84 \\
\hline Regent & can & 8.84 & 109.49 & 1.52 & 71.81 & 6.63 & 129.89 & 3.28 & 1.51 & 22.90 & 5.56 & 2.49 & $*$ \\
\hline Tizian & can & 6.84 & 72.45 & 1.92 & 50.76 & 3.60 & 74.76 & 3.63 & 6.47 & 23.30 & 8.94 & 0.13 & 319.19 \\
\hline $\begin{array}{l}\text { Average for red wine } \\
\text { varieties } \\
\text { Relative Deviation }\end{array}$ & & $\begin{array}{l}10.03 \\
36.16\end{array}$ & & $\begin{array}{c}1.94 \\
34.84\end{array}$ & & $\begin{array}{l}4.97 \\
50.17\end{array}$ & & $\begin{array}{l}3.32 \\
4.63\end{array}$ & & $\begin{array}{c}20.29 \\
8.87\end{array}$ & & $\begin{array}{c}-0.10 \\
-1682.39\end{array}$ & \\
\hline HIGHLIGHTS & & $15<$ & & $2<$ & & $7<$ & & $<3.2$ & & $21<$ & & & $<A B S 100$ \\
\hline
\end{tabular}

